\title{
2-芳基-3,4-二氢异喹啉类植物源抑菌剂的设计合成及构效关系研究
}

\author{
陈 伟*,a 左怀龙 ${ }^{a}$ 李玉新 ${ }^{b}$ 刘 江 ${ }^{a}$ 周先礼 ${ }^{a}$ \\ ( ${ }^{a}$ 西南交通大学生命科学与工程学院 成都 610031) \\ ( ${ }^{b}$ 南开大学元素有机化学国家重点实验室 天津 300071)
}

\begin{abstract}
摘要 植物病原菌严重危及农作物的生产安全. 为了寻找新型的抑菌剂, 通过合理的分子拼接, 将苯甲酸类和苯酚类 药效团引入异喹啉的 2-位，设计并合成了 15 个 2-芳基-3,4-二氢异喹啉衍生物，利用核磁共振和高分辨质谱鉴定了化合 物结构. 初步离体抑菌结果显示, 大部分化合物对 11 种受试植物病原菌都具有中等到显著的抑制活性, 与阳性对照样 (百菌清、多菌灵)相当. 2-(3-乙氧羰基苯基)-3,4-二氢异喹啉溴化盐(4j)对水稻纹枯病菌的 $\mathrm{EC}_{50}$ 为 $3.8495 \mu \mathrm{g} \cdot \mathrm{mL}^{-1}$, 优于 对照样百菌清 $\left(4.6328 \mu \mathrm{g} \cdot \mathrm{mL}^{-1}\right)$. 其中有 5 个化合物对小麦纹枯病菌的 $\mathrm{EC}_{50}\left(7.4583 \sim 15.4495 \mu \mathrm{g} \cdot \mathrm{mL}^{-1}\right)$ 都优于百菌清 (16.0137 $\left.\mu \mathrm{g} \cdot \mathrm{mL}^{-1}\right)$, 其中 2-(4-甲氧羰基苯基)-3,4-二氢异喹啉溴化盐(4f)最为显著, 为植物源抑菌剂的开发提高了理论 基础.
\end{abstract}

关键词 2-芳基-3,4-二氢异喹啉；植物源；抑菌剂；构效关系

\section{Design, Synthesis and Structure-Activity Relationships of Plant-Based 2-Aryl-3,4-dihydroisoquinolin-2-iums as Potential Antifungal Agents}

\author{
Chen, Wei*a Zuo, Huailong $^{a} \quad$ Li, Yuxin $^{b} \quad$ Liu, Jiang $^{a} \quad$ Zhou, Xianli $^{a}$ \\ ( ${ }^{a}$ School of Life Science and Engineering, Southwest Jiaotong University, Chengdu 610031) \\ ( ${ }^{b}$ State Key Laboratory of Elemento-organic Chemistry, Nankai University, Tianjin 300071)
}

\begin{abstract}
In order to discover more potent antifungal, a series of 2-aryl-3,4-dihydroisoquinolin-2-iums were reasonable designed and productive synthesized by introducing benzoic acid and phenol pharmacophores into the 2-position of isoquinoline. Their structures were identified by NMR and HRMS. The preliminary in vitro antifungal results showed that most of the title compounds exhibited moderate to significant inhibitory activities against various phytopathogenic fungi at $50 \mu \mathrm{g} \bullet \mathrm{mL}^{-1}$, and were equal to controls (chlorothalonil, carbendazim). The concentration of 50\% inhibition rate $\left(\mathrm{EC}_{50}\right)$ of 2-(3-ethoxycarbonylphenyl)-3,4-dihydroisoquinolin-2-ium (4j) against $R$. solani was $3.8495 \mu \mathrm{g} \bullet \mathrm{mL}^{-1}$, which was significantly superior to chlorothalonil $\left(4.6328 \mu \mathrm{g} \cdot \mathrm{mL}^{-1}\right)$. All the $\mathrm{EC}_{50}$ values of 5 compounds $\left(7.4583 \sim 15.4495 \mu \mathrm{g} \bullet \mathrm{mL}^{-1}\right)$ against $R$. cerealis were better than chlorothalonil $\left(16.0137 \mu \mathrm{g} \cdot \mathrm{mL}^{-1}\right)$, and 2-(4-methoxycarbonylphenyl)-3,4-dihydroisoquinolin-2-ium (4f) is the best one. The present results provide valuable information for development of plant-based antifungal agents.

Keywords 2-aryl-3,4-dihydroisoquinolin-2-ium; botanical; antifungal activity; structure-activity relationship
\end{abstract}

由植物病原菌引起的植物病害是农作物生产中的 主要危害, 通常会导致农产品产量和质量显著下降，并 造成巨大的经济损失 ${ }^{[1 \sim 3]}$. 同时，一些植物病原菌还能 产生真菌毒素危及人类和动物健康 ${ }^{[4,5]}$. 然而, 植物病原 菌的防治仍是一个难题, 商用化学杀菌剂并没有完全有 效. 此外, 随着农用化学品的普遍应用, 农药残留、农药
抗性和环境污染已成为一个严重问题 ${ }^{[6,7]}$. 因此，开发新 型的抑菌剂来有效控制植物病害具有重要的应用价值.

植物源抑菌剂因环境兼容性好, 对哺乳动物低毒等 特点, 受到了研究者的高度重视 ${ }^{[8,9]}$. 血根碱(Sanguinarine)和白屈菜红碱(Chelerythine)是季铵盐型苯并菲啶生 物碱(QBA $\mathrm{S}_{\mathrm{S}}$ )典型代表(图 1), 具有显著抗微生物病原体

* Corresponding author. E-mail: chenweicstq@163.com

Received May 10, 2019; revised June 2, 2019; published online June 24, 2019

Dedicated to the 100th anniversary of the birth of Professor Ruyu Chen.

Project supported by the National Natural Science Foundation of China (No. 21702173), the Fundamental Research Funds for the Central Universities (No. 2682016CX104) and the Miaozi Project of Scientific and Technological Innovation of Sichuan Province (No. 2018090).

国家自然科学基金(No. 21702173)、中央高校基本科研业务费(No. 2682016CX104)和四川省科技创新苗子工程(No. 2682017QY04)资助项目. 
活性 ${ }^{[10,11]}$. Zhou 等研究指出 $\mathrm{C}=\mathrm{N}^{+}$是 $\mathrm{QBA}_{\mathrm{S}}$ 的关键药效 团，利用骨架跃迁的原理，设计并合成了系列 2-芳基-3, 4-二氢异喹啉衍生物(ADHIQs), 大部分化合物的抑菌 活性优于先导物血根碱和白屈菜碱 ${ }^{[12,13]}$. 因此, ADHIQs 可以作为一个理想的 $\mathrm{QBA}_{\mathrm{S}}$ 模型物, 进行植物源抑菌剂 研究.

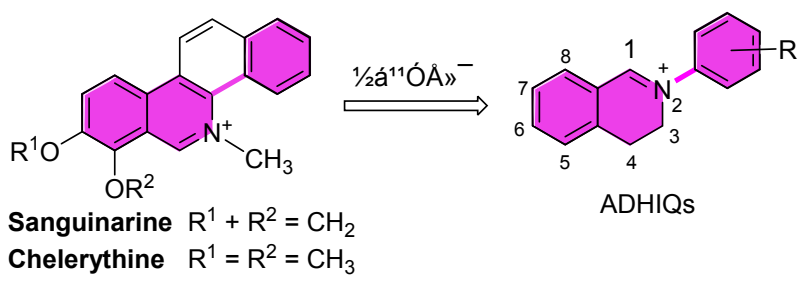

图 1 模型化合物 2-芳基-3,4-二氢异喹啉衍生物(ADHIQs)的 由来

Figure 1 Origin of lead compounds 2-aryl-3,4-dihydroisoquinolin-2-ium (ADHIQs)

苯甲酸类和苯酚类结构单元广泛存在于植物次生 代谢产物中, 也是医药和农用化学品中重要的药效基 才, 氟吗啉、嘧菌酯和申嗪霉素等都是含有这类结构单 元的抑菌剂 ${ }^{[14,15]}$. 由于其显著的药理活性, 针对这类药 效基团的研究一直受到研究者的重视. 为了更加深入的 研究 ADHIQs, 寻找理想的植物源抑菌剂. 本文结合前 期的研究基础, 利用药效团拼接, 将苯甲酸类和苯酚类 药效团引入异喹啉的 $\mathrm{N}$ 位, 设计并合成了一系列 ADHIQs 4a 4o (图 2), 并测试了目标化合物对 11 种植 物病原菌的抑菌活性.

\section{1 结果与讨论}

\section{1 中间体和目标化合物的合成}

本文以异色满 1 为起始原料, 经溴化铜催化一步高
效地制备关键中间体 2-溴乙基苯甲醛(2), 然后与取代 苯胺 3 在冰浴冷却下反应生成活性中间体亚胺，进一步 发生分子内的环合得到目标化合物 $4 \mathbf{a} \sim \mathbf{4 0}$ (Scheme 1). 在 $\mathrm{NMR}$ 谱图中, 存在 $\mathrm{CH}=\mathrm{N}^{+}$的特征信号峰 $\left(\delta_{\mathrm{H}}>9, \mathrm{~s}\right.$, $\left.1 \mathrm{H} ; \delta_{\mathrm{C}}>160\right)$; 经 HRMS 检测, $\mathrm{ESI}^{+}$模式下可见[M$\left.\mathrm{Br}^{-}\right]^{+}$峰, 表明目标化合物为季亚胺异喹啉形式.

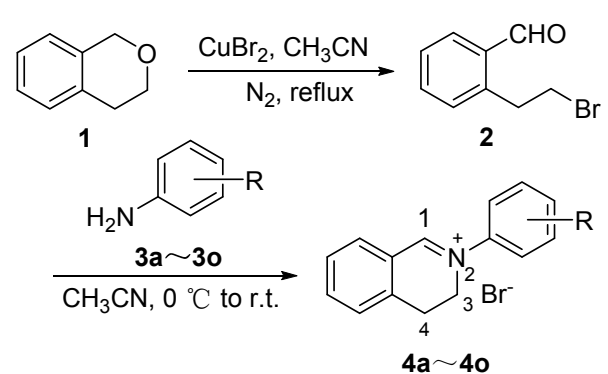

a: $\mathrm{R}=2-\mathrm{OH}-4-\mathrm{Cl} ; \mathbf{b}: \mathrm{R}=2-\mathrm{OH}-4-\mathrm{Br} ; \mathbf{c}: \mathrm{R}=2-\mathrm{OH}-5-\mathrm{NO}_{2}$;

d: $\mathrm{R}=2-\mathrm{OCH}_{3}-4-\mathrm{Br} ; \mathbf{e}: \mathrm{R}=4-\mathrm{COOH} ; \mathbf{f}: \mathrm{R}=4-\mathrm{CO}_{2} \mathrm{CH}_{3}$;

g: $\mathrm{R}=2-\mathrm{CO}_{2} \mathrm{CH}_{3} ; \mathbf{h}: \mathrm{R}=3-\mathrm{CO}_{2} \mathrm{CH}_{3} ; \mathrm{i}: \mathrm{R}=4-\mathrm{CO}_{2} \mathrm{C}_{4} \mathrm{H}_{9}$;

j: $\mathrm{R}=3-\mathrm{CO}_{2} \mathrm{C}_{2} \mathrm{H}_{5} ; \mathbf{k}: \mathrm{R}=3,5-\left(\mathrm{CO}_{2} \mathrm{CH}_{3}\right)_{2} ; \mathrm{I}: \mathrm{R}=2,5-\left(\mathrm{CO}_{2} \mathrm{CH}_{3}\right)_{2}$

m: $\mathrm{R}=2-\mathrm{CO}_{2} \mathrm{C}_{2} \mathrm{H}_{5}, 4-\mathrm{Cl} ; \mathbf{n}: \mathrm{R}=3-\mathrm{OH}-4-\mathrm{CO}_{2} \mathrm{CH}_{3}$;

o: $\mathrm{R}=2-\mathrm{CH}_{3}-4-\mathrm{COOH}$

图式 1 目标化合物 $\mathbf{4 a} \sim \mathbf{4 0}$ 的合成路线

Scheme 1 Synthetic route of title compounds $\mathbf{4 a} \sim \mathbf{4 0}$

\section{2 目标化合物的抑菌活性}

初步离体抑菌结果显示(表 1$)$, 在 $50 \mathrm{mg} \cdot \mathrm{L}^{-1}$ 的处理 浓度下，大部分化合物都具有中等到优异的抑菌活性， 与阳性对照样(百菌清和多菌灵)接近或相当. 对于所有 受试菌种，至少有 4 种目标化合物对其抑制活性大于 $60 \%$. 其中, $4 a \sim 4 c, 4 f$ 和 $4 \mathbf{i} \sim 4 k$ 对 7 种及以上病原菌的 抑制率在 $60.0 \%$ 以上. 尤其是 $4 \mathrm{a}, 4 \mathrm{f}, 4 \mathrm{j}, 4 \mathrm{k}$ 和 $4 \mathrm{n}$ 对水稻 纹枯病菌和小麦纹枯病菌的抑制率都高达 $98.0 \%$ 以上; $4 \mathrm{a}$ 对小麦赤霉病菌和玉米小斑病菌的抑菌活性为 $100.0 \%$ ，与对照样百菌清和多菌灵一致. 对于花生褐斑

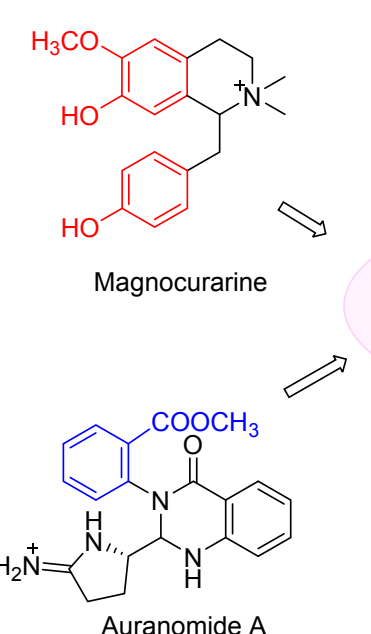

Auranomide A

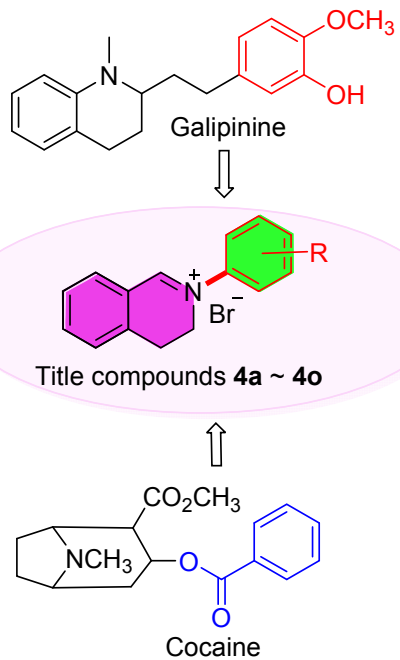

图 2 目标化合物 $\mathbf{4 a} \sim \mathbf{4 0}$ 的设计

Figure 2 Design of title compounds $\mathbf{4 a} \sim \mathbf{4 0}$ 
表 1 目标化合物 $\mathbf{4 a} \sim \mathbf{4 0}$ 的离体抑菌活性 $\left(50 \mathrm{mg} \cdot \mathrm{L}^{-1}, \%\right)$

Table 1 In vitro antifungal activities of target compound $\mathbf{4 a} \sim \mathbf{4 0}\left(50 \mathrm{mg} \cdot \mathrm{L}^{-1}, \%\right)$

\begin{tabular}{cccccccccccc}
\hline No. & $\mathrm{AS}^{a}$ & $\mathrm{FG}$ & $\mathrm{PC}$ & $\mathrm{SS}$ & $\mathrm{RS}$ & $\mathrm{CC}$ & $\mathrm{PP}$ & $\mathrm{FM}$ & $\mathrm{HM}$ & $\mathrm{CL}$ & $\mathrm{RC}$ \\
\hline $\mathbf{4 a}$ & 61.1 & 100.0 & 69.2 & 29.5 & 99.8 & 68.4 & 93.5 & 94.4 & 97.0 & 100.0 & 98.2 \\
$\mathbf{4 b}$ & 35.7 & 75.0 & 41.9 & 21.2 & 68.8 & 63.2 & 90.3 & 94.4 & 97.0 & 96.8 & 86.0 \\
$\mathbf{4 c}$ & 42.9 & 75.0 & 58.1 & 69.2 & 50.0 & 63.2 & 80.6 & 77.8 & 66.7 & 71.0 & 89.5 \\
$\mathbf{4 d}$ & 35.7 & 50.0 & 9.7 & 50.0 & 65.6 & 52.6 & 71.0 & 66.7 & 45.5 & 64.5 & 89.5 \\
$\mathbf{4 e}$ & 22.2 & 10.7 & 88.5 & 29.5 & 12.9 & 60.9 & 64.5 & 33.3 & 42.4 & 41.9 & 80.7 \\
$\mathbf{4 f}$ & 63.6 & 30.8 & 67.7 & 66.7 & 100.0 & 57.1 & 100.0 & 62.5 & 66.7 & 64.7 & 100.0 \\
$\mathbf{4 g}$ & 54.5 & 53.8 & 22.6 & 44.4 & 60.7 & 35.7 & 50.0 & 31.3 & 22.2 & 17.6 & 38.5 \\
$\mathbf{4 h}$ & 36.4 & 69.2 & 45.2 & 50.0 & 71.4 & 50.0 & 73.1 & 56.3 & 55.6 & 35.3 & 92.3 \\
$\mathbf{4 i}$ & 72.7 & 19.2 & 67.7 & 100.0 & 80.4 & 71.4 & 84.6 & 75.0 & 77.8 & 64.7 & 100.0 \\
$\mathbf{4 j}$ & 72.7 & 57.7 & 67.7 & 72.2 & 100.0 & 42.9 & 88.5 & 68.8 & 61.1 & 47.1 & 100.0 \\
$\mathbf{4 k}$ & 72.7 & 46.2 & 54.8 & 55.6 & 100.0 & 64.3 & 92.3 & 68.8 & 66.7 & 35.3 & 100.0 \\
$\mathbf{4 \mathbf { l }}$ & 36.4 & 38.5 & 16.1 & 66.7 & 53.6 & 42.9 & 84.6 & 31.3 & 44.4 & 17.6 & 38.5 \\
$\mathbf{4 m}$ & 27.3 & 23.1 & 9.7 & 27.8 & 53.6 & 7.1 & 19.2 & 25.0 & 22.2 & 5.9 & 38.5 \\
$\mathbf{4 n}$ & 72.7 & 34.6 & 51.6 & 83.3 & 100.0 & 57.1 & 53.8 & 56.3 & 50.0 & 23.5 & 100.0 \\
$\mathbf{4 0}$ & 18.2 & 38.5 & 25.8 & 72.2 & 42.9 & 28.6 & 76.9 & 25.0 & 50.0 & 23.5 & 15.4 \\
CH $^{b}$ & 90.9 & 57.7 & 80.6 & 100.0 & 91.1 & 57.1 & 92.3 & 87.5 & 72.2 & 70.6 & 100.0 \\
CA $^{b}$ & 54.5 & 100.0 & 48.4 & 100.0 & 100.0 & 21.4 & 92.3 & 81.3 & 100.0 & 100.0 & 100.0 \\
\hline
\end{tabular}

${ }^{a}$ AS: Alternaria solani, FG: Fusahum graminearum, PC: Phytophthora capsici, SS: Sclerotinia sclerotiorum, RS: Rhizoctonia solani, CC: Cercosporaara chidicola, PP: Physalospora piricola, FM: Fusarium moniliforme Sheld, HM: Helminthosporium maydis Nisik \& Miy, CL: Colletotrichum lagenarium, RC: Rhizotonia cerealis ${ }^{b} \mathrm{CH}$ : Chlorothalonil, CA: Carbendazim.

表 2 部分化合物对水稻纹枯病菌和小麦纹枯病菌的 $\mathrm{EC}_{50}\left(\mu \mathrm{g} \cdot \mathrm{mL}^{-1}\right)$

Table $2 \mathrm{EC}_{50}\left(\mu \mathrm{g} \cdot \mathrm{mL}^{-1}\right)$ of some compounds against $R$. solani and $R$. cerealis

\begin{tabular}{|c|c|c|c|c|c|c|}
\hline \multirow{2}{*}{ No. } & \multicolumn{3}{|c|}{ R. solani } & \multicolumn{3}{|c|}{ R. cerealis } \\
\hline & $\mathrm{EC}_{50}$ & Toxic regression equation & $r$ & $\mathrm{EC}_{50}$ & Toxic regression equation & $r$ \\
\hline $4 f$ & 5.0256 & $y=2.0389 x+3.5703$ & 0.9709 & 7.4583 & $y=2.4912 x+3.2577$ & 0.9989 \\
\hline $4 \mathbf{i}$ & - & - & - & 10.8475 & $y=2.4888 x+2.6024$ & 0.9984 \\
\hline $4 j$ & 3.8495 & $y=1.5781 x+4.0761$ & 0.9835 & 10.0970 & $y=2.8167 x+2.4458$ & 0.9879 \\
\hline $4 k$ & 9.3223 & $y=2.6780 x+2.4036$ & 0.9730 & 15.4495 & $y=2.7646 x+2.1142$ & 0.9986 \\
\hline $4 n$ & 10.7726 & $y=2.9948 x+1.9084$ & 0.9829 & 13.3171 & $y=2.8105 x+2.1487$ & 0.9976 \\
\hline $\mathrm{CH}^{a}$ & 4.6328 & $y=2.1850 \mathrm{x}+3.5451$ & 0.9933 & 16.0137 & $y=2.5731 x+2.1887$ & 0.9960 \\
\hline
\end{tabular}

${ }^{a} \mathrm{CH}:$ Chlorothalonil.

病菌, $4 \mathbf{a} \sim 4 \mathrm{c}, 4 \mathrm{e}, 4 \mathrm{i}$ 和 $4 \mathrm{k}$ 的抑制率大于 $60.0 \%$, 优于阳 性对照药 $(\leqslant 57.1 \%) ; 4 \mathbf{a}$ 和 $4 \mathrm{~b}$ 对水稻恶苗病菌的活性 (94.4\%)优于对照样 $(\leqslant 87.5 \%)$. 进一步 $\mathrm{EC}_{50}$ 测试结果表 明(表 2), 4f $, 4 \mathbf{j}, 4 \mathbf{k}, 4 \mathbf{n}$ 对水稻纹枯病菌的 $\mathrm{EC}_{50}$ 为 3.8495 $10.7726 \mu \mathrm{g} \bullet \mathrm{mL}^{-1}$, 其中 $\mathbf{4 j}\left(3.8495 \mu \mathrm{g} \bullet \mathrm{mL}^{-1}\right)$ 优 于对照样百菌清 $\left(4.6328 \mu \mathrm{g} \bullet \mathrm{mL}^{-1}\right), \mathbf{4 f}\left(\mathrm{EC}_{50}=5.0256\right.$ $\left.\mu \mathrm{g} \bullet \mathrm{mL}^{-1}\right)$ 与百菌清接近. $4 \mathbf{f}, \mathbf{4 i} \sim \mathbf{4 k}$ 和 $4 \mathbf{n}$ 对小麦纹枯病 菌的 $\mathrm{EC}_{50}\left(7.4583 \sim 15.4495 \mu \mathrm{g} \bullet \mathrm{mL}^{-1}\right)$ 都优于百菌清 $\left(16.0137 \mu \mathrm{g} \bullet \mathrm{mL}^{-1}\right), \mathbf{4 f}$ 最为显著.

与文献 $[12,13,17]$ 对比研究表明, 季亚胺异喹啉是 关键母核, 异喹啉 2-位为苯酚类药效团或者苯甲酸类药 效团时都有助于抑菌活性, 并且苯环上取代基 $\mathrm{R}$ 的种 类、位置对植物病菌的活性和抑菌谱有显著影响. (1)在 苯酚类药效团衍生物中, 含有弱吸电子基团有利于抑菌 活性 [4a (2-OH-5-Cl) $>$ 4b (2-OH-5-Br) $>>4$ c (2-OH-5$\mathrm{NO}_{2}$ )]. (2)在苯甲酸类药效团中, 苯甲酸酯衍生物的活 性优于苯甲酸 $\left[\mathbf{4 f}\left(4-\mathrm{COOCH}_{3}\right) \approx \mathbf{4 i}\left(4-\mathrm{COOC}_{4} \mathrm{H}_{9}\right)>\mathbf{4 e}\right.$
(4-COOH)]. (3)当取代基 $R$ 为单酯时, $R$ 位于苯环的 3/4 位, 有助于抑菌活性 $\left[\mathbf{4 f}\left(4-\mathrm{COOCH}_{3}\right) \approx \mathbf{4 i}\left(4-\mathrm{COOC}_{4}-\right.\right.$ $\left.\left.\mathrm{H}_{9}\right)>\mathbf{4 h}\left(3-\mathrm{COOCH}_{3}\right)>\mathbf{4 g}\left(2-\mathrm{COOCH}_{3}\right)\right]$. (4)当 $\mathrm{R}$ 为单酯 取代基或者双酯时, 抑菌活性无明显区别 [4k $(3,5-$ $\left.\left(\mathrm{COOCH}_{3}\right)_{2}\right)$ 与 $\mathbf{4 h}, \mathbf{4 l}\left(2,5-\left(\mathrm{COOCH}_{3}\right)_{2}\right)$ 与 $\left.\mathbf{4 g}\right]$. (5)酯基中 烷基的大小对活性无显著影响 (4f 与 $4 \mathbf{i}, 4 \mathbf{h}$ 与 $\mathbf{4 j}$ ). 总体 表明, 在异喹啉的 $\mathrm{N}$ 位引入苯酚类和苯甲酸类药效才可 提高化合物的抑菌活性. 在苯酚类药效团衍生物中 $(4 \mathbf{a} \sim 4 \mathbf{d})$, 苯环上有弱吸电基团有利于抑菌活性和抑菌 广谱性; 而对于苯甲酸类药效团衍生物 $(\mathbf{4 e} \sim \mathbf{4 0})$, 取代 基 $\mathrm{R}$ 位于苯环 $3 / 4$ 位更优.

\section{2 结论}

本文通过合理的分子拼接，将苯酚类和苯甲酸类药 效团引入异喹啉的 2-位, 设计并合成了 15 个 2-芳基-3, 4-二氢异喹啉衍生物 $4 \mathbf{a} \sim \mathbf{4 o}$, 利用 NMR 和 HRMS 鉴定 了其结构. 在 $50 \mathrm{mg} \cdot \mathrm{L}^{-1}$ 的测试浓度下, 大部分化合物 
都具有中等到优异的离体抑菌活性, 并且部分化合物与 对照样百菌清和多菌灵相当. $4 a, 4 f, 4 j, 4 k$ 和 $4 n$ 对水稻 纹枯病菌和小麦纹枯病菌的抑制率高达 $98.0 \%$ 以上, 其 中 $4 \mathbf{j}\left(\mathrm{EC}_{50}=3.8495 \mu \mathrm{g} \cdot \mathrm{mL}^{-1}\right)$ 对水稻纹枯病菌的 $\mathrm{EC}_{50}$ 优 于对照样百菌清 $\left(4.6328 \mu \mathrm{g} \bullet \mathrm{mL}^{-1}\right) ; \mathbf{4 f}, \mathbf{4 i} \sim \mathbf{4 k}$ 和 $\mathbf{4 n}$ 对小 麦纹枯病菌的 $\mathrm{EC}_{50}\left(7.4583 \sim 15.4495 \mu \mathrm{g} \bullet \mathrm{mL}^{-1}\right)$ 都优于百 菌清 $\left(16.0137 \mu \mathrm{g} \cdot \mathrm{mL}^{-1}\right), \mathbf{4 f}$ 最为显著. 研究结果表明, 在 异喹啉 2-位引入苯酚类药效团或者苯甲酸类药效团时 都有助于抑菌活性, 为新型植物源抑菌剂的创制提供了 理论基础.

\section{3 实验部分}

\section{1 仪器和试剂}

$600 / 400 \mathrm{MHz}$ 核磁共振仪(Brucker AV 600/400, Bruker), $\mathrm{CDCl}_{3}$ 和 DMSO- $d_{6}$ 为溶剂, TMS 为内标. Xevo G2-S Tof 型高分辨质谱仪(Waters). 所用试剂均为市售 化学纯或分析纯试剂, 用前无需进行处理.

\section{2 化合物的合成}

\subsection{1 中间体 2-溴乙基苯甲醛 2 的合成}

将异色满 1 (0.05 mol, $6.71 \mathrm{~g})$ 和溴化铜 $(0.06 \mathrm{~mol}$, $13.4 \mathrm{~g})$ 溶于 $80 \mathrm{~mL}$ 乙腈中, 氮气保护下加热至回流, 薄 层色谱板(TLC)监测至反应完全. 冷却后减压脱除溶剂, 向残余物中加入 $100 \mathrm{~mL}$ 乙酸乙酯, 饱和食盐水洗涤至 中性, 无水硫酸钠干燥, 柱层析分离得无色液体 $\mathbf{2}^{[16]}$, 产率 92.6\%. ${ }^{1} \mathrm{H}$ NMR (400 $\left.\mathrm{MHz}, \mathrm{CDCl}_{3}\right) \delta: 10.16(\mathrm{~s}, 1 \mathrm{H})$, $7.40 \sim 7.35(\mathrm{~m}, 1 \mathrm{H}), 7.34 \sim 7.25(\mathrm{~m}, 3 \mathrm{H}), 4.01(\mathrm{t}, J=5.7$ $\mathrm{Hz}, 2 \mathrm{H}), 2.90$ (t, $J=5.7 \mathrm{~Hz}, 2 \mathrm{H})$.

\section{2 .2 目标化合物 $\mathbf{4 a} \sim \mathbf{4 0}$ 的合成}

以化合物 $4 \mathbf{a}$ 合成为例. 将 $1.0 \mathrm{mmol}$ 的 2-氨基-4-氯 苯酚 3a 溶于 $10 \mathrm{~mL}$ 乙腈中, 冰浴冷却, 滴加溶有 2 (1.2 $\mathrm{mmol})$ 的乙腈 $(5 \mathrm{~mL})$ 溶液, 摚拌过夜. 减压脱除溶剂, 向 残余物中加入 $10 \mathrm{~mL}$ 乙酸乙酯, 充分搅拌, 抽滤, 固体 用乙酸乙酯洗涤, 烘干得目标化合物 4a. 其余目标化合 物的合成方法与 $4 \mathrm{a}$ 相同, 将反应底物 2-氨基-4-氯苯酚 替换为各种取代的苯胺 $\mathbf{3 b} \sim \mathbf{3 o}$.

2-(4-氯-2-羟基苯基)-3,4-二氢异喹啉溴化盐(4a): 浅褐色固体, 产率 $81.2 \%$. m.p. 216 $219{ }^{\circ} \mathrm{C} ;{ }^{1} \mathrm{H}$ NMR $\left(400 \mathrm{MHz}, \mathrm{DMSO}-d_{6}\right) \delta: 11.36(\mathrm{~s}, 1 \mathrm{H}), 9.61(\mathrm{~s}, 1 \mathrm{H}), 8.01$ $(\mathrm{d}, J=7.5 \mathrm{~Hz}, 1 \mathrm{H}), 7.94 \sim 7.90(\mathrm{~m}, 2 \mathrm{H}), 7.67 \sim 7.59(\mathrm{~m}$, 2H), 7.53 (dd, $J=8.8,2.6 \mathrm{~Hz}, 1 \mathrm{H}), 7.21$ (d, $J=8.9 \mathrm{~Hz}$, $1 \mathrm{H}), 4.41$ (t, $J=7.8 \mathrm{~Hz}, 2 \mathrm{H}), 3.39(\mathrm{t}, J=7.8 \mathrm{~Hz}, 2 \mathrm{H}) ;{ }^{13} \mathrm{C}$ NMR (100 MHz, DMSO- $\left.d_{6}\right) \delta: 170.33,150.31,139.18$, $137.80,135.30,132.30,131.41,128.94,128.83,126.28$, 125.68, 123.10, 119.26, 51.81, 25.42. HRMS (ESI) calcd for $\mathrm{C}_{15} \mathrm{H}_{13} \mathrm{CINO}\left[\mathrm{M}-\mathrm{Br}^{-}\right]^{+}$258.0686, found 258.0680.

2-(4-溴-2-羟基苯基)-3,4-二氢异喹啉溴化盐(4b): 褐色固体，产率 84.1\%. m.p. 210 213 ${ }^{\circ} \mathrm{C} ;{ }^{1} \mathrm{H}$ NMR (400 MHz, DMSO- $\left.d_{6}\right) \delta$ : $11.40(\mathrm{~s}, 1 \mathrm{H}), 9.59(\mathrm{~s}, 1 \mathrm{H}), 8.01$ (dd, $J=8.4,5.0 \mathrm{~Hz}, 2 \mathrm{H}), 7.91(\mathrm{t}, J=7.4 \mathrm{~Hz}, 1 \mathrm{H}), 7.67 \sim 7.57$ $(\mathrm{m}, 3 \mathrm{H}), 7.18 \sim 7.13(\mathrm{~m}, 1 \mathrm{H}), 4.40(\mathrm{t}, J=7.8 \mathrm{~Hz}, 2 \mathrm{H}), 3.38$ $(\mathrm{t}, J=7.8 \mathrm{~Hz}, 2 \mathrm{H}) ;{ }^{13} \mathrm{C} \mathrm{NMR}\left(100 \mathrm{MHz}, \mathrm{DMSO}-d_{6}\right) \delta$ : $170.30,150.74,139.17,137.80,135.29,135.15,131.84$, $128.97,128.93,128.83,125.68,119.68,110.25,51.81$, 25.42. HRMS (ESI) calcd for $\mathrm{C}_{15} \mathrm{H}_{13} \mathrm{BrNO}\left[\mathrm{M}-\mathrm{Br}^{-}\right]^{+}$ 302.0181, found 302.0190.

2-(2-差基-4-硝基苯基)-3,4-二氢异喹啉溴化盐(4c): 褐色固体, 产率 79.6\%. m.p. 234 236 ${ }^{\circ} \mathrm{C} ;{ }^{1} \mathrm{H}$ NMR (400 MHz, DMSO- $\left.d_{6}\right) \delta: 9.57(\mathrm{~s}, 1 \mathrm{H}), 8.78(\mathrm{~d}, J=2.4 \mathrm{~Hz}, 1 \mathrm{H})$, 8.35 (dd, $J=9.1,2.7 \mathrm{~Hz}, 1 \mathrm{H}), 8.00$ (d, $J=7.5 \mathrm{~Hz}, 1 \mathrm{H})$, $7.90(\mathrm{t}, J=7.4 \mathrm{~Hz}, 1 \mathrm{H}), 7.64 \sim 7.59(\mathrm{~m}, 2 \mathrm{H}), 7.37$ (d, $J=$ $9.2 \mathrm{~Hz}, 1 \mathrm{H}), 4.41(\mathrm{t}, J=7.7 \mathrm{~Hz}, 2 \mathrm{H}), 3.38(\mathrm{t}, J=7.7 \mathrm{~Hz}$, $2 \mathrm{H}) ;{ }^{13} \mathrm{C}$ NMR (100 MHz, DMSO- $\left.d_{6}\right) \delta: 157.83,139.69$, $138.95,137.91,135.14,130.89$, 128.98, 128.79, 127.89, 125.99, 122.83, 122.77, 117.62, 51.39, 25.40. HRMS (ESI) calcd for $\mathrm{C}_{15} \mathrm{H}_{13} \mathrm{~N}_{2} \mathrm{O}_{3}\left[\mathrm{M}-\mathrm{Br}^{-}\right]^{+}$269.0926, found 269.0926.

2-(4-溴-2-甲氧基苯基)-3,4-二氢异喹啉溴化盐(4d): 浅褐色固体, 产率 89.6\%. m.p. 201 203 ${ }^{\circ} \mathrm{C} ;{ }^{1} \mathrm{H} \mathrm{NMR}$ $\left(400 \mathrm{MHz}, \mathrm{DMSO}-d_{6}\right) \delta: 9.58(\mathrm{~s}, 1 \mathrm{H}), 8.01(\mathrm{~d}, J=7.5 \mathrm{~Hz}$, $1 \mathrm{H}), 7.92(\mathrm{td}, J=7.6,1.1 \mathrm{~Hz}, 1 \mathrm{H}), 7.78(\mathrm{~d}, J=8.4 \mathrm{~Hz}$, $1 \mathrm{H}), 7.69 \sim 7.57(\mathrm{~m}, 3 \mathrm{H}), 7.46(\mathrm{dd}, J=8.4,1.9 \mathrm{~Hz}, 1 \mathrm{H})$, 4.38 (t, $J=7.8 \mathrm{~Hz}, 2 \mathrm{H}), 3.97$ (s, 3H), 3.39 (t, $J=7.8 \mathrm{~Hz}$, $2 \mathrm{H}) ;{ }^{13} \mathrm{C}$ NMR (100 MHz, DMSO- $\left.d_{6}\right) \delta: 170.43,153.44$, $139.25,137.86,135.34,131.30,128.96,128.85,127.99$, $125.70,125.63,124.45,117.16,57.75,52.06,25.42$. HRMS (ESI) calcd for $\mathrm{C}_{16} \mathrm{H}_{15} \mathrm{BrNO}\left[\mathrm{M}-\mathrm{Br}^{-}\right]^{+} 316.0337$, found 316.0350 .

2-(4-羧基苯基)-3,4-二氢异喹啉溴化盐(4e)：浅黄色 固体, 产率 69.8\%. m.p. $211 \sim 214{ }^{\circ} \mathrm{C} ;{ }^{1} \mathrm{H}$ NMR (400 $\left.\mathrm{MHz}, \mathrm{DMSO}-d_{6}\right) \delta$ : $13.40(\mathrm{br}, 1 \mathrm{H}), 9.80(\mathrm{~s}, 1 \mathrm{H}), 8.20$ (d, $J=8.6 \mathrm{~Hz}, 2 \mathrm{H}), 8.08$ (d, $J=7.4 \mathrm{~Hz}, 1 \mathrm{H}), 8.02$ (d, $J=8.2$ $\mathrm{Hz}, 2 \mathrm{H}), 7.91(\mathrm{t}, J=7.4 \mathrm{~Hz}, 1 \mathrm{H}), 7.65 \sim 7.61(\mathrm{~m}, 2 \mathrm{H}), 4.63$ $(\mathrm{t}, J=7.8 \mathrm{~Hz}, 2 \mathrm{H}), 3.44(\mathrm{t}, J=7.9 \mathrm{~Hz}, 2 \mathrm{H}) ;{ }^{13} \mathrm{C} \mathrm{NMR}(100$ $\left.\mathrm{MHz}, \mathrm{DMSO}-d_{6}\right) \delta: 168.37,166.62,146.34,139.21$, $137.84,135.63,133.09,131.26,128.80,126.04,123.62$, 51.09, 25.32. HRMS (ESI) calcd for $\mathrm{C}_{16} \mathrm{H}_{14} \mathrm{NO}_{2}[\mathrm{M}-$ $\left.\mathrm{Br}^{-}\right]^{+}$252.1025, found 252.1042.

2-(4-甲氧羰基苯基)-3,4-二氢异喹啉溴化盐(4f)：黄 色固体, 产率 $91.2 \%$. m.p. 191 $193{ }^{\circ} \mathrm{C} ;{ }^{1} \mathrm{H}$ NMR (400 
MHz, DMSO- $\left.d_{6}\right) \delta: 9.80(\mathrm{~s}, 1 \mathrm{H}), 8.24(\mathrm{~d}, J=8.7 \mathrm{~Hz}, 2 \mathrm{H})$, $8.09 \sim 8.04(\mathrm{~m}, 3 \mathrm{H}), 7.93(\mathrm{t}, J=7.3 \mathrm{~Hz}, 1 \mathrm{H}), 7.67 \sim 7.62$ (m, 2H), $4.64(\mathrm{t}, J=7.8 \mathrm{~Hz}, 2 \mathrm{H}), 3.93(\mathrm{~s}, 3 \mathrm{H}), 3.45$ (t, $J=$ $7.8 \mathrm{~Hz}, 2 \mathrm{H}) ;{ }^{13} \mathrm{C}$ NMR $\left(100 \mathrm{MHz}, \mathrm{DMSO}-d_{6}\right) \delta: 168.54$, $165.60,146.61,139.27,137.88,135.68,131.81,131.17$, $128.80,126.02,125.95,123.78,53.15,51.07,25.32$. HRMS (ESI) calcd for $\mathrm{C}_{17} \mathrm{H}_{16} \mathrm{NO}_{2}\left[\mathrm{M}-\mathrm{Br}^{-}\right]^{+}$266.1181, found 266.1204 .

2-(2-甲氧羰基苯基)-3,4-二氢异喹啉溴化盐(4g)：黄 色固体, 产率 60.3\%. m.p. $144 \sim 146{ }^{\circ} \mathrm{C} ;{ }^{1} \mathrm{H}$ NMR (400 MHz, DMSO- $\left.d_{6}\right) \delta: 9.85(\mathrm{~s}, 1 \mathrm{H}), 8.49(\mathrm{dd}, J=7.9,0.8 \mathrm{~Hz}$, $1 \mathrm{H}), 8.36(\mathrm{~d}, J=7.1 \mathrm{~Hz}, 1 \mathrm{H}), 8.18(\mathrm{dd}, J=7.9,1.4 \mathrm{~Hz}$, $1 \mathrm{H}), 7.82(\mathrm{td}, J=7.8,1.5 \mathrm{~Hz}, 1 \mathrm{H}), 7.74(\mathrm{td}, J=7.6,1.1 \mathrm{~Hz}$, $1 \mathrm{H}), 7.65$ (td, $J=7.8,1.0 \mathrm{~Hz}, 1 \mathrm{H}), 7.48(\mathrm{t}, J=7.6 \mathrm{~Hz}, 1 \mathrm{H})$, 7.39 (d, $J=7.6 \mathrm{~Hz}, 1 \mathrm{H}), 4.42$ (br, 2H), 3.89 (s, 3H), 3.61 (br, $2 \mathrm{H}) ;{ }^{13} \mathrm{C}$ NMR $\left(100 \mathrm{MHz}, \mathrm{CDCl}_{3}\right) \delta: 168.56,164.32$, $142.45,138.79,136.75,136.45,135.43,132.22,131.51$, $128.72,128.19,128.01,125.30,124.00,53.98,53.03$, 26.20. HRMS (ESI) calcd for $\mathrm{C}_{17} \mathrm{H}_{16} \mathrm{NO}_{2}\left[\mathrm{M}-\mathrm{Br}^{-}\right]^{+}$ 266.1181, found 266.1204.

2-(3-甲氧羰基苯基)-3,4-二氢异喹啉溴化盐(4h): 黄 色固体, 产率 $80.9 \%$. m.p. $184 \sim 186{ }^{\circ} \mathrm{C} ;{ }^{1} \mathrm{H}$ NMR $(400$ $\left.\mathrm{MHz}, \mathrm{CDCl}_{3}\right) \delta: 10.37(\mathrm{~s}, 1 \mathrm{H}), 8.57(\mathrm{dd}, J=8.1,1.7 \mathrm{~Hz}$, $1 \mathrm{H}), 8.44(\mathrm{~d}, J=7.4 \mathrm{~Hz}, 1 \mathrm{H}), 8.37$ (s, 1H), 8.14 (d, $J=7.9$ $\mathrm{Hz}, 1 \mathrm{H}), 7.75(\mathrm{td}, J=7.6,1.0 \mathrm{~Hz}, 1 \mathrm{H}), 7.66(\mathrm{t}, J=8.0 \mathrm{~Hz}$, $1 \mathrm{H}), 7.48 \sim 7.41(\mathrm{~m}, 1 \mathrm{H}), 4.65(\mathrm{t}, J=7.9 \mathrm{~Hz}, 2 \mathrm{H}), 3.92(\mathrm{~s}$, $3 \mathrm{H}), 3.57(\mathrm{t}, J=7.9 \mathrm{~Hz}, 2 \mathrm{H}) ;{ }^{13} \mathrm{C} \mathrm{NMR}\left(100 \mathrm{MHz}, \mathrm{CDCl}_{3}\right)$ $\delta: 166.69,165.22,142.58,139.05,136.79,136.45,132.00$, $131.81,130.94,128.83,128.18,127.87,125.67,123.03$, 52.78, 51.81, 25.92. HRMS (ESI) calcd for $\mathrm{C}_{17} \mathrm{H}_{16} \mathrm{NO}_{2}$ $\left[\mathrm{M}-\mathrm{Br}^{-}\right]^{+}$266.1181, found 266.1204.

2-(4-丁氧羰基苯基)-3,4-二氢异喹啉溴化盐(4i): 黄 色固体, 产率 87.4\%. m.p. $155 \sim 157{ }^{\circ} \mathrm{C} ;{ }^{1} \mathrm{H}$ NMR $(400$ $\left.\mathrm{MHz}, \mathrm{CDCl}_{3}\right) \delta: 10.48(\mathrm{~s}, 1 \mathrm{H}), 8.39$ (d, $\left.J=7.4 \mathrm{~Hz}, 1 \mathrm{H}\right)$, $8.18(\mathrm{~d}, J=8.9 \mathrm{~Hz}, 2 \mathrm{H}), 8.12(\mathrm{~d}, J=8.8 \mathrm{~Hz}, 2 \mathrm{H}), 7.74 \sim$ $7.67(\mathrm{~m}, 1 \mathrm{H}), 7.46 \sim 7.33(\mathrm{~m}, 2 \mathrm{H}), 4.63(\mathrm{t}, J=7.9 \mathrm{~Hz}, 2 \mathrm{H})$, $4.32(\mathrm{t}, J=6.6 \mathrm{~Hz}, 2 \mathrm{H}), 3.51(\mathrm{t}, J=7.9 \mathrm{~Hz}, 2 \mathrm{H}), 1.80 \sim$ $1.70(\mathrm{~m}, 2 \mathrm{H}), 1.55 \sim 1.40(\mathrm{~m}, 2 \mathrm{H}), 0.98(\mathrm{t}, J=7.4 \mathrm{~Hz}, 3 \mathrm{H})$; ${ }^{13} \mathrm{C}$ NMR $\left(100 \mathrm{MHz}, \mathrm{CDCl}_{3}\right) \delta: 166.66,164.90,145.23$, 139.26, 136.97, 136.65, 132.74, 131.51, 128.85, 128.20, 125.61, 123.05, 65.59, 51.76, 30.68, 25.93, 19.27, 13.79 . HRMS (ESI) calcd for $\mathrm{C}_{20} \mathrm{H}_{22} \mathrm{NO}_{2}\left[\mathrm{M}-\mathrm{Br}^{-}\right]^{+} 308.1651$, found 308.1660 .

2-(3-乙氧羰基苯基)-3,4-二氢异喹啉溴化盐(4j): 黄 色固体, 产率 $84.1 \%$. m.p. $182 \sim 185{ }^{\circ} \mathrm{C} ;{ }^{1} \mathrm{H}$ NMR (400
$\left.\mathrm{MHz}, \mathrm{CDCl}_{3}\right) \delta: 10.38(\mathrm{~s}, 1 \mathrm{H}), 8.62(\mathrm{dd}, J=8.1,1.7 \mathrm{~Hz}$, $1 \mathrm{H}), 8.46(\mathrm{~d}, J=7.4 \mathrm{~Hz}, 1 \mathrm{H}), 8.32$ (s, 1H), 8.16 (d, $J=7.9$ $\mathrm{Hz}, 1 \mathrm{H}), 7.75$ (dt, $J=7.6,3.8 \mathrm{~Hz}, 1 \mathrm{H}), 7.67$ (t, $J=8.0 \mathrm{~Hz}$, 1H), $7.48(\mathrm{t}, J=7.6 \mathrm{~Hz}, 1 \mathrm{H}), 7.42(\mathrm{~d}, J=7.6 \mathrm{~Hz}, 1 \mathrm{H}), 4.63$ (t, $J=7.9 \mathrm{~Hz}, 2 \mathrm{H}), 4.40$ (q, $J=7.1 \mathrm{~Hz}, 2 \mathrm{H}), 3.57$ (t, $J=7.9$ $\mathrm{Hz}, 2 \mathrm{H}), 1.42(\mathrm{dd}, J=7.1 \mathrm{~Hz}, 3 \mathrm{H}) ;{ }^{13} \mathrm{C}$ NMR $(100 \mathrm{MHz}$, $\left.\mathrm{CDCl}_{3}\right) \delta: 166.71,164.78,142.62,139.08,136.85,136.46$, $132.42,131.83,130.97,128.89,128.22,127.84,125.71$, $122.95,61.96,51.86,25.97,14.34$. HRMS (ESI) calcd for $\mathrm{C}_{18} \mathrm{H}_{18} \mathrm{NO}_{2}\left[\mathrm{M}-\mathrm{Br}^{-}\right]^{+}$280.1338, found 280.1354.

2-(3,5-二甲氧羰基苯基)-3,4-二氢异喹啉澳化盐 (4k): 黄色固体, 产率 91.1\%. m.p. $193 \sim 195{ }^{\circ} \mathrm{C} ;{ }^{1} \mathrm{H}$ NMR (400 MHz, $\left.\mathrm{CDCl}_{3}\right) \delta: 10.37(\mathrm{~s}, 1 \mathrm{H}), 8.81$ (d, $J=1.1$ $\mathrm{Hz}, 2 \mathrm{H}), 8.76$ (s, 1H), 8.39 (d, $J=7.6 \mathrm{~Hz}, 1 \mathrm{H}), 7.76$ (t, $J=$ $7.5 \mathrm{~Hz}, 1 \mathrm{H}), 7.54 \sim 7.39(\mathrm{~m}, 2 \mathrm{H}), 4.64(\mathrm{t}, J=7.8 \mathrm{~Hz}, 2 \mathrm{H})$, 3.95 (s, 6H), 3.60 (t, $J=7.8 \mathrm{~Hz}, 2 \mathrm{H}) ;{ }^{13} \mathrm{C}$ NMR $(100 \mathrm{MHz}$, $\left.\mathrm{CDCl}_{3}\right) \delta: 167.22,164.56,143.15,139.15,136.77,136.65$, $132.77,132.32,128.87,128.27,127.88,125.86,53.04$, 51.99, 26.00. HRMS (ESI) calcd for $\mathrm{C}_{19} \mathrm{H}_{18} \mathrm{NO}_{4}[\mathrm{M}-$ $\left.\mathrm{Br}^{-}\right]^{+}$324.1236, found 324.1247.

2-(2,5-二甲氧羰基苯基)-3,4-二氢异喹啉溴化盐(41): 白色固体, 产率 79.5\%. m.p. 196 198 ${ }^{\circ} \mathrm{C} ;{ }^{1} \mathrm{H}$ NMR (400 $\left.\mathrm{MHz}, \mathrm{CDCl}_{3}\right) \delta: 10.32(\mathrm{~s}, 1 \mathrm{H}), 8.78(\mathrm{~s}, 1 \mathrm{H}), 8.45(\mathrm{~d}, J=$ $7.6 \mathrm{~Hz}, 1 \mathrm{H}), 8.30(\mathrm{~s}, 2 \mathrm{H}), 7.79(\mathrm{t}, J=7.5 \mathrm{~Hz}, 1 \mathrm{H}), 7.51(\mathrm{t}$, $J=7.5 \mathrm{~Hz}, 1 \mathrm{H}), 7.44(\mathrm{~d}, J=7.6 \mathrm{~Hz}, 1 \mathrm{H}), 4.44(\mathrm{br}, 2 \mathrm{H})$, 3.95 (s, 3H), 3.94 (s, 3H), 3.63 (br, 2H); ${ }^{13} \mathrm{C}$ NMR $(100$ $\left.\mathrm{MHz}, \mathrm{CDCl}_{3}\right) \delta: 169.64,164.38,163.68,142.31,139.21$, $136.99,136.56,135.99,132.73,132.29,128.87,128.35$, $128.25,128.12,125.29,53.88,53.55,53.18,26.15$. HRMS (ESI) calcd for $\mathrm{C}_{19} \mathrm{H}_{18} \mathrm{NO}_{4}\left[\mathrm{M}-\mathrm{Br}^{-}\right]^{+} 324.1236$, found 324.1247.

2-(4-氯-2-乙氧羰基苯基)-3,4-二氢异喹啉溴化盐 (4m): 白色固体, 产率 76.5\%. m.p. $185 \sim 187{ }^{\circ} \mathrm{C} ;{ }^{1} \mathrm{H}$ NMR (400 MHz, $\left.\mathrm{CDCl}_{3}\right) \delta$ : 9.97 (s, 1H), $8.61(\mathrm{~s}, 1 \mathrm{H}), 8.36$ (d, $J=7.3 \mathrm{~Hz}, 1 \mathrm{H}), 8.15$ (d, $J=8.4 \mathrm{~Hz}, 1 \mathrm{H}), 7.76$ (t, $J=7.4$ $\mathrm{Hz}, 1 \mathrm{H}), 7.62(\mathrm{~d}, J=8.3 \mathrm{~Hz}, 1 \mathrm{H}), 7.55 \sim 7.38(\mathrm{~m}, 2 \mathrm{H})$, 4.37 (br, 2H), 4.34 (q, J=7.1 Hz, 2H), 3.64 (br, 2H), 1.34 (t, $J=7.1 \mathrm{~Hz}, 3 \mathrm{H}) ;{ }^{13} \mathrm{C}$ NMR $\left(100 \mathrm{MHz}, \mathrm{CDCl}_{3}\right) \delta: 169.08$, $163.01,142.92,140.81,139.07,136.70,133.32,131.65$, $128.60,128.40,128.36,127.97,125.11,123.02,62.53$, 53.98, 26.13, 14.05. HRMS (ESI) calcd for $\mathrm{C}_{18} \mathrm{H}_{17} \mathrm{ClNO}_{2}$ $\left[\mathrm{M}-\mathrm{Br}^{-}\right]^{+}$314.0948, found 314.0956.

2-(3-差基-4-甲氧羰基苯基)-3,4-二氢异喹啉溴化盐 (4n): 黄色固体，产率 $74.7 \%$. m.p. $150 \sim 152{ }^{\circ} \mathrm{C} ;{ }^{1} \mathrm{H}$ NMR (600 MHz, DMSO- $\left.d_{6}\right) \delta: 10.85$ (s, 1H), $9.74(\mathrm{~s}, 1 \mathrm{H})$, 
$8.09 \sim 8.01(\mathrm{~m}, 2 \mathrm{H}), 7.91(\mathrm{~s}, 1 \mathrm{H}), 7.68 \sim 7.58(\mathrm{~m}, 2 \mathrm{H})$, $7.57 \sim 7.37(\mathrm{~m}, 2 \mathrm{H}), 4.58(\mathrm{~s}, 2 \mathrm{H}), 3.93(\mathrm{~s}, 3 \mathrm{H}), 3.41(\mathrm{~s}$, $2 \mathrm{H}) ;{ }^{13} \mathrm{C}$ NMR (150 MHz, DMSO- $\left.d_{6}\right) \delta: 168.50,168.16$, $160.44,147.82$, 139.29, 137.95, 135.72, 135.10, 132.36, $128.78,125.95,116.17,113.95,112.38,53.31,50.90$, 25.30. HRMS (ESI) calcd for $\mathrm{C}_{17} \mathrm{H}_{16} \mathrm{NO}_{3}\left[\mathrm{M}-\mathrm{Br}^{-}\right]^{+}$ 282.1130, found 282.1149.

2-(4-羧基-2-甲基苯基)-3,4-二氢异喹啉溴化盐(4o): 白色固体，产率 81.3\%. m.p. 280 282 ${ }^{\circ} \mathrm{C} ;{ }^{1} \mathrm{H}$ NMR (400 MHz, DMSO- $\left.d_{6}\right) \delta: 9.62(\mathrm{~s}, 1 \mathrm{H}), 8.08(\mathrm{~s}, 1 \mathrm{H}), 8.05 \sim 8.01$ (m, 2H), 7.95 (t, $J=7.6 \mathrm{~Hz}, 1 \mathrm{H}), 7.88(\mathrm{~d}, J=8.2 \mathrm{~Hz}, 1 \mathrm{H})$, $7.66(\mathrm{t}, J=7.0 \mathrm{~Hz}, 2 \mathrm{H}), 4.46(\mathrm{t}, J=7.9 \mathrm{~Hz}, 2 \mathrm{H}), 3.48$ (t, $J=7.9 \mathrm{~Hz}, 2 \mathrm{H}), 2.52(\mathrm{~s}, 3 \mathrm{H}) ;{ }^{13} \mathrm{C}$ NMR $(100 \mathrm{MHz}$, DMSO- $\left.d_{6}\right) \delta: 170.71,166.72,145.78,139.30,137.81$, $135.44,133.54,133.31,133.16,128.96,128.79,128.76$, $126.29,125.75,52.06,25.35,17.65$. HRMS (ESI) calcd for $\mathrm{C}_{17} \mathrm{H}_{16} \mathrm{NO}_{2}\left[\mathrm{M}-\mathrm{Br}^{-}\right]^{+}$266.1181, found 266.1204.

\section{3 化合物的生物活性测试}

\subsection{1 抑菌活性测试}

用平血法 ${ }^{[1]}$ 测试了目标化合物 $\mathbf{4 a} \sim \mathbf{4 0}$ 对番茄早疫 病菌(A. solani), 小麦赤霉病菌 (F. graminearum), 辣椒 疫霉病菌 ( P. capsici), 油菜菌核病菌 $(S$. sclerotiorum), 水 稻纹枯病菌 $(R$. solani), 花生褐斑病菌 (C. chidicola), 苹 果轮纹病菌 (P. piricola), 水稻恶苗病菌 (F. moniliforme), 玉米小斑病菌( H. maydis Nisik \& Miy), 西瓜炭疽病菌 (C. lagenarium) 和小麦纹枯病菌(R. cerealis) 共计 11 种常 见病菌的离体抑菌活性, 百菌清和多菌灵为阳性对照 药. 在无菌条件下, 将化合物溶于二甲基亚砜中, 配成 浓度为 $3.0 \times 10^{4} \mathrm{mg} \cdot \mathrm{L}$ 的溶液, 用吐温溶液稀释成浓度 $500 \mathrm{mg} \cdot \mathrm{L}$ 的测试液. 取 $1 \mathrm{~mL}$ 上述溶液加入到 $9 \mathrm{~mL}$ 马铃 薯葡萄糖琼脂(PDA)培养基中, 待培养基完全凝固后接 入供试菌种, 设置空白对照, 实验重复 3 次. 在 $25 \pm$ $1{ }^{\circ} \mathrm{C}$ 下培养 $72 \mathrm{~h}$ 后, 测量菌落直径, 并与空白对照组进 行比较得到抑制率.
抑制率 $/ \%=($ 空白对照菌落增长直径一药剂处理菌落 增长直径)/空白对照菌落增长直径 $\times 100 \%$

抑制中浓度 $\left(\mathrm{EC}_{50}\right)$ 的测定：根据上述方法，采用等 倍稀释, 分别测试浓度为 $25,12.5,6.25,3.125$ 与 $1.56 \mu \mathrm{g}$ • $\mathrm{mL}^{-1}$ 时的抑制率, 然后用软件 SPSS 计算抑制中浓度 $\mathrm{EC}_{50}$.

辅助材料(Supporting Information) 目标化合物 $\mathbf{4 a} \sim$ 40 的离体抑菌活性. ${ }^{1} \mathrm{H} \mathrm{NMR},{ }^{13} \mathrm{C} \mathrm{NMR}$ 和 HRMS 图谱. 这些材料可以免费从本刊网站(http://sioc-journal. cn/)上 下载.

\section{References}

[1] Chen, W.; Li, Y. X.; Zhou, Y. Y.; Ma, Y.; Li, Z. M. Chin. Chem. Lett. 2019, DOI: 10.1016/j.cclet.2019.04.072.

[2] Liang, R.; Li, X.; Yuan, W.; Jin, S.; Hou, S.; Wang, M.; Wang, H. J. Agric. Food Chem. 2018, 66, 9907

[3] Hou, Z.; Zhu, L. F.; Yu, X. C.; Sun, M. Q.; Miao, F.; Zhou, L. J. Agric. Food Chem. 2016, 64, 2847.

[4] Eskola, M.; Altieri, A.; Galobart, J. World Mycotoxin J. 2018, 11, 277.

[5] Wu, Y. L.; Wang, D. L.; Guo, E. H.; Song, S.; Feng, J. T.; Zhang, X. Bioorg. Med. Chem. Lett. 2017, 27, 1284.

[6] Moss, S.; Ulber, L.; den Hoed, I. Crop Prot. 2019, 115, 13.

[7] Velioglu, Y. S.; Fikirdesici-Ergen, S.; Aksu, P.; Altindag, A. Tarim Bilim. Derg. 2018, 24, 245.

[8] Wei, Y. X.; Xu, X. Y.; Song, X. Curr. Org. Chem. 2017, 21, 1907.

[9] Mehta, S.; Sharma, K. Int. J. Pharma Sci. Res. 2016, 7, 4327.

[10] Ping, G.; Wang, Y.; Shen, L.; Wang, Y.; Hu, X.; Chen, J.; Hu, B.; Cui, L.; Meng, Q.; Li, C. Chem. Commun. 2017, 53, 7381

[11] Ling, F.; Wu, Z. Q.; Jiang, C.; Liu, L.; Wang, G. X. J. Fish Dis. 2016, 39, 993

[12] Lei, P.; Xu, Y.; Du, J.; Yang, X. L.; Yuan, H. Z.; Xu, G. F.; Ling, Y. Bioorg. Med. Chem. Lett. 2016, 26, 2544.

[13] Yang, R.; Gao, Z. F.; Zhao, J. Y.; Li, W. B.; Zhou, L.; Miao, F. J. Agric. Food Chem. 2015, 63, 1906.

[14] Thebault, M.; Mueller, U.; Kandelbauer, A.; Zikulnig-Rusch, E.; Lammer, H. Eur. J. Wood Wood Prod. 2017, 75, 853.

[15] Liu, C. L.; Yang, J. C. Handbook of Modern Pesticide, Chemical Industry Press, Beijing, 2017 (in Chinese). (刘长令, 杨吉春, 现代农药手册, 化学工业出版社, 北京, 2017.)

[16] Zhou, M. Y.; Kong, S. S.; Zhang, L. Q.; Zhao, M.; Duan, J. A.; Ou-yang, Z.; Wang, M. Tetrahedron Lett. 2013, 54, 3962.

[17] Ma, Y. N.; Yang, X. J.; Pan, L.; Hou, Z.; Geng, H. L.; Song, X. P.; Miao, F.; Zhou, L. Chem. Pharm. Bull. 2013, 61, 204.

(Li, L.; Fan, Y.) 